BRIEF COMMUNICATION

This is an open access article distributed under the terms of the Creative Commons BY-NC-ND Licence

\title{
Arabidopsis KIN gamma subunit 1 has a potential to regulate activity of sucrose nonfermenting 1-related protein kinase $2 \mathrm{~s}(\mathrm{SnRK} 2 \mathrm{~s})$ in vitro
}

\author{
M. PUNKKINEN ${ }^{1}$, K. DENESSIOUK ${ }^{1,2}$, and H. FUJII ${ }^{1 *}$ \\ Molecular Plant Biology Unit, Department of Biochemistry, University of Turku, Turku, Finland ${ }^{1}$ \\ Faculty of Science and Engineering, Åbo Akademi University, Turku, Finland ${ }^{2}$
}

\begin{abstract}
Plants must precisely regulate their signalling pathways to respond to environmental changes promptly. Sucrose non fermenting1 (SNF1)-related protein kinases (SnRK) 2 are essential kinases in abiotic stress responses, including responses to abscisic acid. Although homologs of SnRKs in yeast require a $\gamma$-subunit for full activation, it has been unclear whether SnRK2s in higher plants are affected by $\gamma$-subunits. In this report, we aimed to show the effect of Arabidopsis KIN $\gamma$ subunit 1 (KING1), which is a potential $\gamma$-subunit, on the activity of SnRK2. A recombinant KING1 bound to SnRK2.6 and functionally inhibited its activity in vitro. On the other hand, KING1 facilitated the activity of SnRK2.2. Structural models suggest that significant structural changes occurred as a result of KING1 binding to the C-terminal tail of SnRK2s. Since KING1 inhibited the kinase activity of a chimeric protein consisting of the N-terminal domain of SnRK2.6 and the C-terminal domain of SnRK2.2, regulation by KING1 was determined by the N-terminal domain of SnRK2s. Together, these results show that KING1 can mediate activity of SnRK2s in vitro.
\end{abstract}

Addditional key words: abscisic acid, activation of kinase, structural models.

As sessile organisms, plants, have to use their signal transduction pathways to acclimate to harsh environmental conditions such as reduced availability of nutrients and water. Pathway components, such as kinases, must be regulated precisely to achieve optimal responses. The SNF1/AMP-activated protein kinases (AMPKs) are an important kinase family involved in the regulation of energy depletion in yeasts and mammals (Hardie 2011). For full activation, SNF1 forms a heterotrimeric protein complex with the $\beta$-subunit and $\gamma$-subunit (SNF4) (Celenza and Carlson 1989). In Arabidopsis thaliana, the closest homologs of SNF1 are SNF1-related protein kinases (SnRK) 1: SnRK1.1 and 1.2 (also named KIN10 and 11). As was shown in yeast two-hybrid assays, SnRK1s can bind to three $\beta$-subunits (AKIN $\beta$ 1, 2, and 3) and two $\gamma$-subunits: KIN $\gamma$ subunit 1 (KING1; Bouly et al. 1999) and AtSNF4; the latter also contains a $\beta$-subunit-like domain (Kleinow et al. 2000, Lumbreras et al. 2001, Gissot et al. 2006, Bitrián et al. 2011). Meanwhile, AtSNF4, but not KING1, can make a complex with the $\gamma$ - and $\beta$-subunits in co-immunoprecipitation assay (Emanuelle et al. 2015). While AtSNF4 has been identified as the functional SnRK1 $\gamma$-subunit, KING1 cannot complement the growth defect of a yeast snf4 mutant on non-glucose plates and might not be involved in SnRK1 signaling (Ramon et al. 2013). Therefore, KING1 might be involved in cellular regulation via pathways other than those that involve SnRK1s. In addition to SnRK1s, plants have two other subfamilies of SnRKs: SnRK2 and SnRK3. The involvement of $\gamma$-subunits in the activation of each of these subfamilies is unclear. The SnRK2s have 10 members in A. thaliana, namely, SnRK2.1-SnRK2.10 (Hrabak et al. 2003). All SnRK2s in A. thaliana, apart from SnRK2.9, are activated by osmotic stress (Boudesocq

Submitted 27 December 2017, last revision 20 July 2018, accepted 1 August 2018.

Abbreviations: ABA - abscisic acid; AMPK - AMP-activated protein kinase; AREB - ABA-responsive element binding factor; GST glutathione S-transferase; KING1 - KIN $\gamma$ subunit 1; MBP - maltose-binding protein; PP2C - protein phosphatase 2C; SnRK - SNF1related protein kinase.

Acknowledgements: We thank the Turku Collegium for Science and Medicine and the Academy of Finland (Project numbers 259169, 263853, 271832, 292763, and 307335) for funding, and the Scandinavian Plant Physiology Society for a travel grant.

*Corresponding author; e-mail: hiroaki.fujii@utu.fi 
A
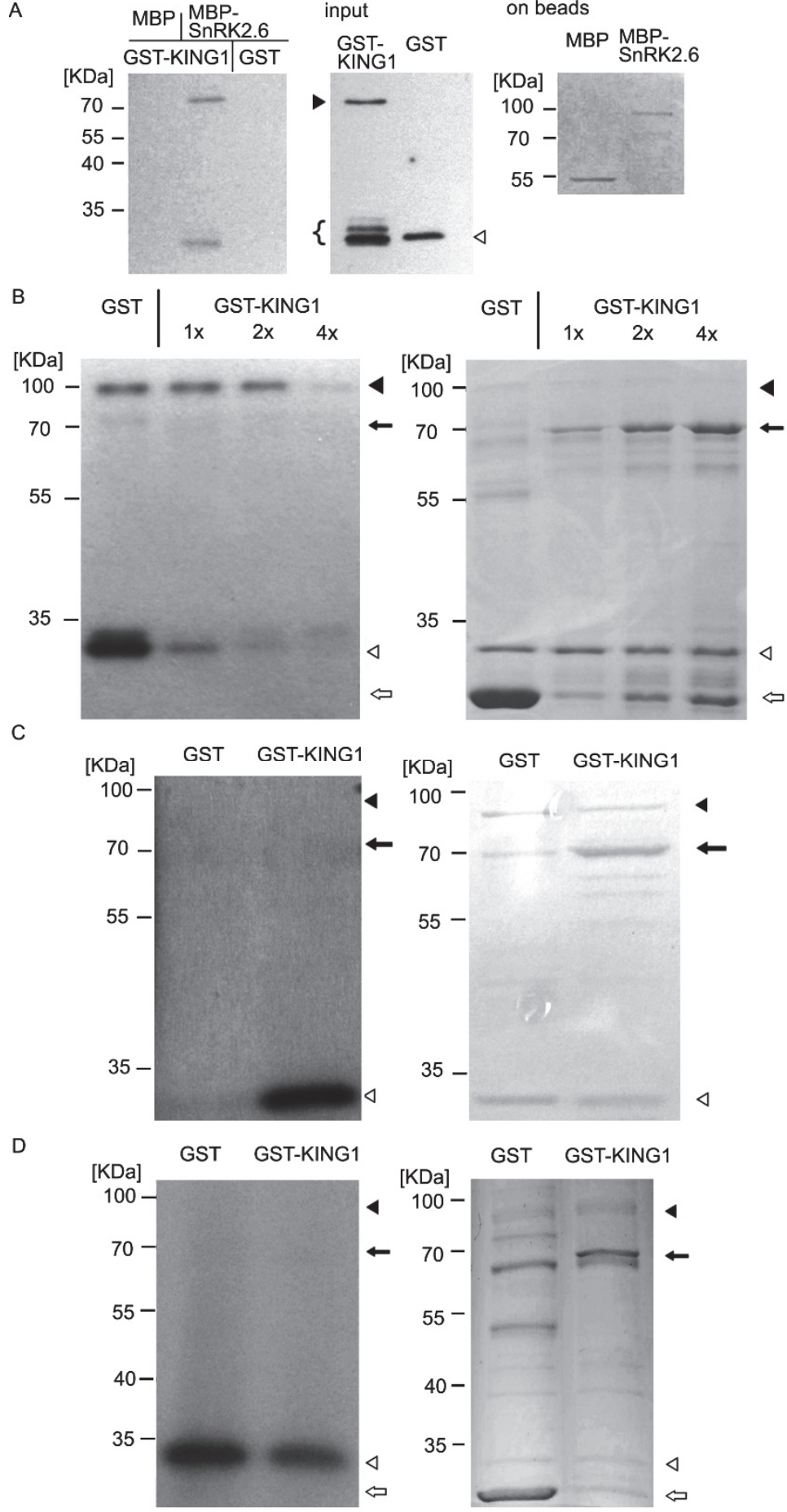

Fig. 1. KIN $\gamma$ subunit 1 (KING1) binds to SnRK2.6 in vitro $(A)$ and affects autophosphorylation and substrate phosphorylation of SnRK2.6 $(B)$, SnRK2.2 $(C)$, and chimeric SnRK2.2-2.6 $(D)$ in different ways. Binding and kinase assays were performed with recombinant proteins produced in Escherichia coli. For binding assay $(A)$, amylose beads with maltose binding protein (MBP) or MBPfused SnRK2.6 were incubated with glutathione S-transferase (GST) or GST-fused KING1. Proteins in the bound fraction (left) and in the input fraction (middle, $10 \%$ ) were detected using an anti-GST-antibody. Coomassie staining was used to show the amounts of proteins on the beads (right). Expected sizes of GST-KING1 and GST are indicated by black and white arrowheads, respectively. Some GST-KING1s were degraded (a curly bracket). For kinase assays $(B-D)$, MBP-fused SnRK2s were incubated with either GST (a white arrow) or GST-fused KING1 (a black arrow) in the presence of $\left[\gamma^{32} \mathrm{P}\right]$ ATP. A GST-fused AREB1a was used as a phosphorylation substrate for SnRK2s. Autophosphorylation (a black arrowhead) and substrate phosphorylation (a white arrowhead) were detected by autoradiography (left). Coomassie staining was used to show the amount of proteins (right). The GST ran out from the bottom of the gel in the SnRK2.2 experiment. 
et al. 2004). Three of the A. thaliana SnRK2s, SnRK2.2, SnRK2.3, and SnRK2.6, are also strongly activated by abscisic acid (ABA; Boudsocq et al. 2004), which is an important factor in stress responses and during plant development such as seed maturation, dormancy, and seedling growth (Leung and Giraudat 1998, Sato et al. 2009, Umezawa et al. 2010, Joshi-Saha et al. 2011). The SnRK2s are one of the core components of the ABAsignalling pathway with an $\mathrm{ABA}$ receptor pyrabactin resistance1/PYR1-like (PYR/PYL) and protein phosphatase 2Cs (PP2Cs) (Fujii et al. 2009, Ma et al. 2009, Park et al. 2009). In the absence of ABA, the PP2C binds to an SnRK2 and inactivates it by preventing selfphosphorylation of the activation loop ( $\mathrm{Ng}$ et al. 2011,
Yunta et al. 2011, Soon et al. 2012), whereas in the presence of ABA, the inhibition of PP2Cs by PYR/PYLs releases SnRK2s from suppression, allowing them to be activated through phosphorylation (Fujii et al. 2009, Umezawa et al. 2009, Vlad et al. 2009). Thus, modification of SnRK2 activity directly affects ABA responses. Papdi et al. (2008) showed in a large-scale screening that overexpression of KING1 leads to ABAinsensitivity during germination. Involvement of KING1 in the ABA pathway and its homology to the $\gamma$-subunit of members of the SNF1 family suggest that KING1 directly regulates the activity of SnRK2s. In this study we analyzed the functional interaction between SnRK2s and KING1 in vitro.

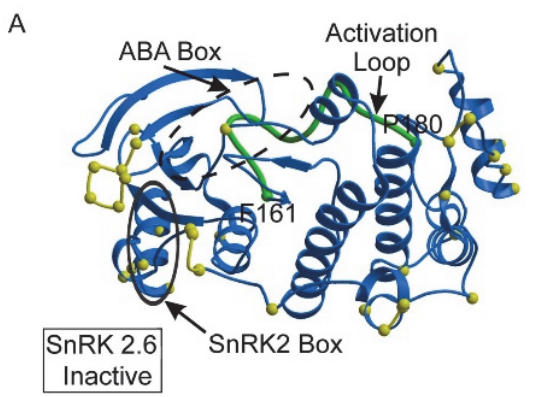

B
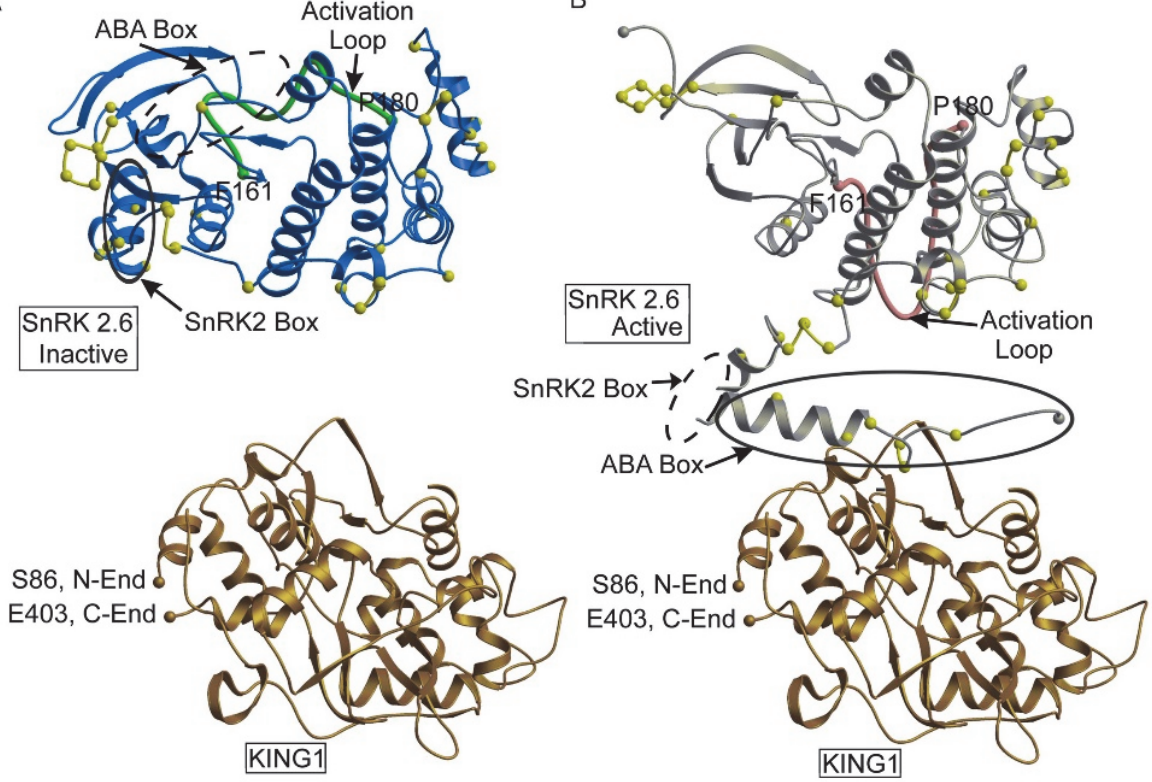

Fig. 2. A structural model of an "activated" SnRK2.6/KING1 complex in comparison with an "inactive" SnRK2.6. $A$ - A model of KING1 (gold, based on the $\gamma-1$ subunit of AMPK, PDB ID: 4CFE:E) in a complex with the "inactive form" of SnRK2.6 (blue, PDB ID: 3UC4). $B$ - A model of KING1 (gold, the same as in $A$ ) in a complex with the "activated form" of SnRK2.6 (grey, based on the AMPK structure PDB ID: 4CFE:A). Amino acids F161 and P180 show ends of the SnRK 2.6 activation loop (green in $A$, pink in $B$ ). In $A$, the position of the SnRK2 box and the estimated position of the ABA box (not present in 3UC4) are shown. In $B$, the estimated position of the SnRK2 box (not present in 4CFE:A) and the position of the ABA box are also indicated. The N-terminal 86 amino acids of KING1 could not be modeled. The putative PP2C binding site is positioned on the "top" of the inactive form of SnRK2.6 structure across the activation loop. Yellow structures indicate different amino acid residues between SnRK2.2 and SnRK2.6.

Firstly, we analyzed whether KING1 binds to SnRK2.6. The GST-KING1, but not GST, was pulled down with MBP-SnRK2.6, but not with maltose-binding protein (MBP) indicating that KING1 binds directly to SnRK2.6 (Fig. 1A). Next, to determine whether KING1 has any effect on the activity of SnRK2.6, we performed an in vitro kinase assay using recombinant proteins. A GST-fused AREB1a was used as a substrate of SnRK2s (Furihata et al. 2006). Compared to GST, GST-KING1 prevented both the autophosphorylation and the substrate phosphorylation of SnRK2.6. Intensities of the major AREB1a band and the SnRK2.6 auto-phosphorylation band decreased in correlation to the amount of KING1 (Fig. 1B) indicating that KING1 inhibited the activity of SnRK2.6 in vitro. Besides, SnRK2.6, SnRK2.2, and SnRK2.3 were classified as ABA-activated SnRK2s. To determine whether the effects of KING1 on SnRK2.6 activities are consistent among ABA-activated SnRK2s, we performed an in vitro kinase assay using recombinant MBP-fused SnRK2.2. Interestingly, SnRK2.2 incubated with GST-KING1 had a higher substrate phosphorylation capacity than SnRK2.2 incubated with GST (Fig. 1C). These results indicate that KING1 facilitates activity of SnRK2.2 in vitro and that SnRK2s can be controlled by 
KING1 in various ways.

Additional plant proteins, such as the $\beta$-subunit, were not assumed to exist in the above in vitro assays. To speculate on how SnRK2s could interact with KING1, we created a molecular model of the SnRK2.6/KING1 complex, which is based on the crystal structure of the full length human AMPK (PDB ID: 4CFE), a heterotrimeric complex between AMPK subunits $\alpha-2$ (4CFE:A), $\beta-1$, and $\gamma-1$ (4CFE:E). The SnRK2.6 sequence was aligned with the AMPK catalytic subunit $\alpha-2$ with a $32 \%$ sequence identity (a $45 \%$ sequence similarity, Fig. 1 Suppl.), and the KING1 sequence was aligned with AMPK subunit $\gamma-1$ with a $15 \%$ sequence identity (a $29 \%$ sequence similarity, Fig. 2 Suppl.). Two consecutive C-terminal helix/loop regions of the SnRK2 structure, the SnRK2 box (Gln303Val317 in SnRK2.6) and the ABA box (Leu333-Met362 in SnRK2.6, Fig. 1 Suppl.), are important for its activation (Yoshida et al. 2006, $\mathrm{Ng}$ et al. 2011). The $\alpha$-helix in the ABA box has a well-defined hydrophobic surface Gly331Leu333-Ile335-Met339-Leu343 pointing inside, towards the core of the structure, and its polar surface Asp334Asp336-Asp337-Asp338-Glu340-Glu341-Asp342-Glu344Asp346 pointing outside serve for interaction with other proteins (Yoshida et al. 2006, $\mathrm{Ng}$ et al. 2011). The structure of 4CFE contains two small molecules bound to the N-terminal domain of the catalytic $\alpha$ subunit, an ATP analogue, and a small molecule activator, a benzimidazole derivative (991) (Xiao et al. 2013). Thus, the model contains the SnRK2.6 structure based on the activatorinduced active form of the catalytic $\alpha$ subunit (Fig. 2B). Interaction of SnRK2.6 and KING1 occurred between the ABA box in the C-terminal tail of SnRK2.6 and KING1 segments (Gln231-Leu244 and Va195-Ala104) (Fig. 2B and Fig. 1 Suppl.). This C-terminal tail, including both the SnRK2 box and the ABA box, is similar in SnRK2.6 and SnRK2.2 (Fig. 1 Suppl.).

Additionally, at least two A. thaliana SnRK2.6 structures have been reported in its inactive form. One is the monomeric and ligand-free structure of the A. thaliana SnRK2.6 with D59A/E60A mutations, which were introduced for the crystallization (PDB ID: $3 \mathrm{UC4}$; $\mathrm{Ng}$ et al. 2011). The second is the structure of the SnRK2.6 bound with PP2C (PDB ID: 3UJG, Soon et al. 2012). The two SnRK2.6 structures (3UC4 and 3UJG) are conformationally similar. When this inactive form is superposed to the model of the SnRK2.6/KING1 complex shown above, the interacting $\mathrm{C}$-terminal tail is turned away from the SnRK2.6/KING1 interface towards the Nterminal active site (Fig. $2 A$ ). The conformational change of the activation loop (Phe161-Pro180 in SnRK2.6) is significant: it points towards the SnRK2.6/KING1 interface in the active form of SnRK2.6 (Fig. 2B), whereas it is flipped towards the active site in the inactive form (Fig. 2A). Except for the C-terminal tail and the activation loop, there is no difference between the active and inactive models. These data suggest that KING1-binding to the C-terminal region induces conformational changes in the structure of SnRK2, causing SnRK2 to adopt the activatorbound conformation. This could explain how KING1 facilitates the activity of SnRK2.2 (Fig. 1C) in the same manner as $\gamma$-subunits positively regulate both SNF1 and SnRK1s kinases (Hardie 2011, Ramon et al. 2013). On the other hand, KING1 inhibits the activity of SnRK2.6 (Fig. $1 B$ ) even though the structural model suggests that the binding sites are located in similar places in the C-terminal regions of the SnRK2s.

To identify the critical domain of SnRK2s in terms of regulation by KING1, we produced a chimeric protein consisting of the N-terminal (1-312) part of SnRK2.6 and the C-terminal (315-362) part of SnRK2.2 (Fig. 1 Suppl.) and tested the effects of KING1 on its activity in in vitro kinase assay. The chimeric SnRK2.6-2.2 protein can be expressed and purified despite being there more degradation bands than in SnRK2.6 or 2.2 (Fig. 3 Suppl.). The chimeric SnRK2.6-2.2 protein reacted similarly to SnRK2.6 with a reduced substrate phosphorylation capacity after treatment with GST-KING1 (Fig. 1D). Although the molecular mechanism is unclear, this result indicates that the effects of KING1 on SnRK2s are not exclusively determined by the C-terminal tail. Multiple pieces of evidence suggest that the $\mathrm{N}$-terminal region is important to this effect: Even though SnRK2.6 and SnRK2.2 have high similarities, several amino acids are different between them (Fig. 2 and Fig. 1 Suppl.). As shown by West et al. (2013), even the small differences in the N-terminal domains of SnRK2.6 and SnRK2.3 (which has $91 \%$ identity to SnRK2.2) result in different stabilities and conformations of the proteins. The SnRK2.6 is also a much more stable protein than SnRK2.2 and SnRK2.3 in the thermal shift assay ( $\mathrm{Ng}$ et al. 2011). We speculate that KING1 binds in a similar manner to SnRK2.2 and SnRK2.6, but cannot induce the same conformational change in SnRK2.6 as the one which activates SnRK2.2. Rather, the binding of KING1 may have an effect on the flexibility of SnRK2.6. This includes the molecular motion of the activation loop, which may happen in SnRK2.6 without KING1 and produce the basal activity of SnRK2.6. There are, however, also other possibilities for interaction between KING1 and SnRK2s. Because the N-terminal end structure of the AMPK $\gamma$-subunit has not been reported, the $\mathrm{N}$-terminal end of KING1 cannot be modelled. The Nterminal end is large enough and close enough to the interphase with SnRK2 to possibly affect the specificity of this protein as both an inhibitor and an activator. The physiological role of KING-mediated inhibition/ facilitation of SnRK2s, including the interaction with $\mathrm{PP} 2 \mathrm{C}$ in vivo, remains to be clarified in future experiments, whereas the results in this study show that KING1 had the potential to affect activities of SnRK2s and played a role in regulation of other kinases besides SnRK1s. 


\section{References}

Bitrián, M., Roodbarkelari, F., Horváth, M., Koncz, C.: BACrecombineering for studying plant gene regulation: developmental control and cellular localization of SnRK1 kinase subunits. - Plant J. 65: 829-842, 2011.

Boudsocq, M., Barbier-Brygoo, H., Laurière, C.: Identification of nine sucrose nonfermenting 1-related protein kinases 2 activated by hyperosmotic and saline stress in Arabidopsis thaliana. - J. biol. Chem. 279: 41758-41766, 2004.

Bouly, J.-P., Gissot, L., Lessard, P., Kreis, M., Thomas, M.: Arabidopsis thaliana proteins related to the yeast SIP and SNF4 interact with AKIN $\alpha 1$, an SNF1-like protein kinase. Plant J. 18: 541-550, 1999.

Celenza, J.L., Carlson, M.: Mutational analysis of the Saccharomyces cerevisiae SNF1 protein kinase and evidence for functional interaction with the SNF4 protein. - Mol. cell. Biol. 9: 5034-5044, 1989.

Emanuelle, S., Hossain, M.I., Moller, I.E., Pedersen, H.L., Van de Meene, A.M.L., Doblin, M.S., Koay, A., Oakhill, J.S., Scott, J.W., Willats, W.G., Kemp, B.E., Bacic, A., Gooley, P.R., Stapleton, D.I.: SnRK1 from Arabidopsis thaliana is an atypical AMPK. - Plant J. 82: 183-192, 2015.

Fujii, H., Chinnusamy, V., Rodrigues A., Rubio S., Antoni R., Park, S.-Y. et al.: In vitro reconstitution of an ABA signaling pathway. - Nature 462: 660-664, 2009.

Furihata, T., Maruyama, K., Fujita, Y., Umezawa, T., Yoshida, R., Shinozaki, K., Yamaguchi-Shinozaki, K.: Abscisic aciddependent multisite phosphorylation regulates the activity of a transcription activator AREB1. - Proc. nat. Acad. Sci. USA 103: 1988-1993, 2006.

Gissot, L., Polge, C., Jossier, M., Girin, T., Bouly, J.-P., Kreis, M., Thomas, M.: AKIN $\beta \gamma$ contributes to SnRK1 heterotrimeric complexes and interacts with two proteins implicated in plant pathogen resistance through its KIS/GBD sequence. - Plant Physiol. 142: 931-944, 2006.

Hardie, D.G.: AMP-activated protein kinase: an energy sensor that regulates all aspects of cell function. - Genes Dev. 25: 1895$1908,2011$.

Hrabak, E.M., Chan, C.W.M., Gribskov, M., Harper, J.F., Choi, J.H., Halford, N, Kudla, J., Luan, S., Nimmo, H.G., Sussman, M.R., Thomas, M., Walker-Simmons, K., Zhu, J.-K., Harmon, A.C.: The Arabidopsis CDPK-SnRK superfamily of protein kinases. - Plant Physiol. 132: 666-680, 2003.

Joshi-Saha, A., Valon, C., Leung, J.: A brand new START: abscisic acid perception and transduction in the guard cell. Sci. Signal. 4 (201): re4, 2011.

Kleinow, T., Bhalerao, R., Breuer, F., Umeda, M., Salchert, K., Koncz, C.: Functional identification of an Arabidopsis Snf4 ortholog by screening for heterologous multicopy suppressors of snf4 deficiency in yeast. - Plant J. 23: 115-122, 2000.

Leung, J., Giraudat, J.: Abscisic acid signal transduction. - Annu. Rev. Plant Physiol. Plant mol. Biol. 49: 199-222, 1998.

Lumbreras, M.M.A., Kleinow, T., Koncz, C., Pagès, M.: Domain fusion between SNF1-related kinase subunits during plant evolution. - EMBO Rep. 2: 55-60, 2001.

Ma, Y., Szostkiewicz, I., Korte, A., Moes, D., Yang, Y., Christmann, A., Grill, E.: Regulators of PP2C phosphatase activity function as abscisic acid sensors. - Science 324: 10641068, 2009

Ng, L.-M., Soon, F.-F., Zhou, X.E., West, G.M., Kovach, A., Suino-Powell, K.M., Chalmers, M.J., Li, J., Yong, E.-L., Zhu, J.-K., Griffin, P.R., Melcher, K., Xu, H.E.: Structural basis for basal activity and autoactivation of abscisic acid (ABA) signaling SnRK2 kinases. - Proc. nat. Acad. Sci. USA 108: 21259-21264, 2011

Papdi, C., Ábrahám, E., Joseph, M.P., Popescu, C., Koncz, C., Szabados, L.: Functional identification of Arabidopsis stress regulatory genes using the controlled cDNA overexpression system. - Plant Physiol. 147: 528-542, 2008.

Park, S.-Y., Fung, P., Nishimura, N., Jensen, D.R., Fujii, H., Zhao, Y., Lumba, S., Santiago, J., Rodrigues, A., Chow, T.F., Alfred, S.E., Bonetta, D., Finkelstein, R., Provart, N.J., Desveaux, D., Rodrigues, P.L., McCourt, P., Zhu, J.-K., Schroeder, J.I., Volkman, B.F., Cutler, S.R.: Abscisic acid inhibits type 2C protein phosphatases via the PYR/PYL family of START proteins. - Science 324: 1068-1071, 2009.

Ramon, M., Ruelens, P., Li, Y., Sheen, J., Geuten, K., Rolland, F.: The hybrid four-CBS-Domain KIN $\beta \gamma$ subunit functions as the canonical $\gamma$ subunit of the plant energy sensor SnRK1. - Plant J. 75: 11-25, 2013.

Sato, A., Sato, Y., Fukao, Y., Fujiwara, M., Umezawa, T., Shinozaki, K.. Hibi, T., Taniguchi, M., Miyake, H., Goto, D.B., Uozumi, N.: Threonine at position 306 of the KAT1 potassium channel is essential for channel activity and is a target site for ABA-activated SnRK2/OST1/SnRK2.6 protein kinase. Biochem. J. 424: 439-448, 2009.

Soon, F.F., Ng, L.M., Zhou, X.E., West, G.M., Kovach, A., Tan, M.H., Suino-Powell, K.M., He, Y., Xu, Y., Chalmers, M.J., Brunzelle, J.S., Zhang, H., Yang, H., Jiang, H., Li, J., Yong, E.L., Cutler, S., Zhu, J.-K., Griffin, P.R., Melcher, K., Xu, H.E.: Molecular mimicry regulates ABA signaling by SnRK2 kinases and PP2C phosphatases. - Science 335: 85-88, 2012.

Umezawa, T., Nakashima, K., Miyakawa, T., Kuromori, T., Tanokura, M., Shinozaki, K., Yamaguchi-Shinozaki, K.: Molecular basis of the core regulatory network in ABA responses: sensing, signaling and transport. - Plant Cell Physiol. 51: 1821-1839, 2010

Vlad, F., Rubio, S., Rodrigues, A., Sirichandra, C., Belin, C., Robert, N., Leung, J., Rodriguez, P.L., Laurière, C., Merlot, S.: Protein phosphatases $2 \mathrm{C}$ regulate the activation of the SNF1related kinase OST1 by abscisic acid in Arabidopsis. - Plant Cell 21: 3170-3184, 2009.

West, G.M., Pascal, B.D., Ng, L.-M., Soon, F.-F., Melcher, K., Xu, H.E, Chalmers, M.J., Griffin, P.R.: Protein conformation ensembles monitored by HDX reveal a structural rationale for abscisic acid signaling protein affinities and activities. Structure 21: 229-235, 2013.

Xiao, B., Sanders, M.J., Carmena, D., Bright, N.J., Haire, L.F., Underwood, E., Patel, B.R., Heath, R.B., Walker, P.A., Hallen, S., Giordanetto, F., Martin, S.R., Carling, D., Gamblin, S.J.: Structural basis of AMPK regulation by small molecule activators. - Nat. Commun. 4: 3017, 2013.

Yoshida, R., Umezawa, T., Mizoguchi, T., Takahashi, S., Takahashi, F., Shinozaki, K.: The regulatory domain of SRK2E/OST1/SnRK2.6 interacts with ABI1 and integrates abscisic acid (ABA) and osmotic stress signals controlling stomatal closure in Arabidopsis. - J. biol. Chem. 281: 53105318, 2006.

Yunta, C., Martinez-Ripoll, M., Albert, A.: SnRK2.6/OST1 from Arabidopsis thaliana: cloning, expression, purification, crystallization and preliminary X-ray analysis of $\mathrm{K} 50 \mathrm{~N}$ and D160A mutants. - Acta. Crystallogr. Sect. F. Struct. Biol. Cryst. Commun. 67: 364-368, 2011. 